\title{
Factores que influyen en la liquidez de las Mypes en tiempos de pandemia por la covid-19
}

\author{
Oscar Apaza Apaza \\ contabilidad.ospaid@gmail.com \\ https://orcid.org/0000-0002-7389-8742 \\ Universidad Peruana Unión - Perú \\ Pablo de la Cruz Marín Apaza \\ pablo.marin@coopcm.com \\ https://orcid.org/0000-0002-2285-9292 \\ Universidad Peruana Unión - Perú \\ Idel Iban Cutipa Ticona \\ ivancontador2009@gmail.com \\ https://orcid.org/0000-0001-6472-4428 \\ Universidad Peruana Unión - Perú
}

\section{RESUMEN}

Las decisiones metodológicas y operacionales se sustentan en información de calidad, ello coadyuva a la obtención y mantenimiento de la liquidez en los emprendimientos. Partiendo de esta premisa, el objetivo del presente artículo fue determinar los factores que influyen en la liquidez de las Micro y Pequeñas Empresas - Mypes, en épocas de la Covid-19. En la muestra se trabajó con 70 empresarios de la plaza Túpac Amaru de la ciudad de Juliaca, cuyas edades oscilan entre los 20 a más de 50 años. A través de una investigación básica, de enfoque cuantitativo, de tipo correlacional y de diseño no experimental, se utilizó un cuestionario. Los resultados fueron una incidencia positiva considerable de $(\mathrm{r}=0,758)$ y un valor de $(\mathrm{p}=0,000)$. Se concluye que los factores control de operaciones $(r=0,438)$, registro de ingresos $(r=0,857)$ y registro de gastos $(r=0,933)$, influyen en la variable liquidez, existiendo una influencia positiva débil del factor estados financieros $(r=0,267)$. Se recomienda incidir en la práctica de la llevanza de los registros de control de las operaciones, con la intención de promover y salvaguardar la liquidez de las Mypes.

Palabras clave: liquidez de las mypes; control de operaciones; registro de ingresos; registro de gastos; estados financieros. 


\title{
Factors influencing the liquidity of Mypes in times \\ of pandemic the covid-19
}

\begin{abstract}
Methodological and operational decisions are based on quality information, which helps to obtain and maintain liquidity in the enterprises. Based on this premise, the objective of this article is to determine the factors that influence the liquidity of Micro and Small Enterprises - Mypes in times of Covid-19. For the sample we worked with 70 entrepreneurs of the Túpac Amaru square in the city of Juliaca, whose ages range from 20 to more than 50 years old. Through a basic research, quantitative approach, correlational type and non-experimental design. A questionnaire was used. The results were a considerable positive incidence of $(r=0.758)$ and a value of $(p=0.000)$. It is concluded that the factors control of operations $(r=0.438)$, recording of income $(r=0.857)$ and recording of expenses $(r=0.933)$, influence the liquidity variable, with a weak positive influence of the factor financial statements $(\mathrm{r}=0.267)$. It is recommended that the practice of keeping records to control operations be encouraged in order to promote and safeguard the liquidity of Mypes.
\end{abstract}

Keywords: liquidity of mypes; control of operations; recording of income; recording of expenses; financial statements.

Artículo recibido: 02 noviembre. 2021 Aceptado para publicación: 28 noviembre 2021 Correspondencia: contabilidad.ospaid@gmail.com

Conflictos de Interés: Ninguna que declarar 


\section{INTRODUCCIÓN}

La epidemia de la COVID-19 está impactando negativamente sobre las actividades económicas, los ingresos de los emprendedores se han vuelto insuficientes a fin de honrar los pagos comprometidos, ello eleva los niveles de vulnerabilidad financiera. En ese contexto, los microempresarios tuvieron menor capacidad de afrontar una parada abrupta de sus actividades, la misma que mermó sus ingresos (Bárcena, 2020).

Al respecto, Torres y Fernández (2021) refieren que la recuperación económica tardará hasta el 2023, inclusive hasta el 2024. Por su parte, Torres et al. (2020) sostiene que la pandemia ha llevado a los hogares, a umbrales por debajo de lo que se define como pobreza relativa, dejándolos inhabilitados y hacer frente a imprevistos. En ese contexto, Carbó (2020) refiere que el papel del estado será trascendental a fin de reactivar la economía, a través de la cadena de pagos o flexibilizando sus acreencias.

En el Perú, el $92.7 \%$ de las empresas son Mypes, y su funcionamiento ha permitido generar impactos positivos en la economía del país; estas mismas son perjudicadas por el aislamiento social obligatorio, las medidas de restricción impuestas por el Estado peruano, las obligaciones tributarias y demás, se suman a los inconvenientes que hasta ahora tienen las Mypes.

El presente trabajo aborda la problemática de qué factores influyen en la liquidez de las Mypes de un sector de comerciantes de la denominada Plaza Túpac Amaru de la ciudad de Juliaca, en el año 2021, por consiguiente, el problema de investigación fue ¿En qué medida los factores que influyen en la liquidez de las Mypes?, con la finalidad de comprender en contexto, la realidad que pasa la unidad de análisis. A efectos de lograr el objetivo de determinar los factores que influyen en la liquidez de las Mypes, se aplicaron cuestionarios a los comerciantes y se revisó literatura especializada, y se estableció un marco teórico que sustenta al presente estudio.

\section{ESTRATEGIAS METODOLÓGICAS O MATERIALES Y MÉTODOS}

La presente investigación, en concordancia con su naturaleza y sus objetivos, fue una investigación de enfoque cuantitativo, de tipo correlacional, de diseño no experimental, transversal. Por cuanto se utiliza el conocimiento pre existente en un propósito concreto, porque no se manipularán las variables de estudio. En el enfoque cuantitativo, según Torres (2016), el investigador se plantea hipótesis, pero sus planteamientos no son tan específicos, y las preguntas investigativas, no siempre se conceptualizan. 
Según Ramos (2020) las investigaciones correlacionales plantean hipótesis y buscan relaciones entre dos o más variables. Por su parte, Barco (2021) refiere que el objetivo de los diseños transversales es conocer la realidad a través de la aplicación de un instrumento en un solo momento temporal.

Las investigaciones no experimentales están basadas en categorías, variables o contextos que se presentan sin la intervención del investigador, éste no altera el objeto, porque solamente se observan los acontecimientos de la manera cómo éstos se manifiestan en su contexto natural, para ser analizados con posterioridad (Hernández y Mendoza, 2018).

La población estuvo constituida por los 85 Mypes de la Asociación de Comerciantes Minoristas de la Plaza Internacional Túpac Amaru - ACOMITA II, del sector de venta de prendas de vestir. Al respecto Hernández (2018) refiere que la población es el conglomerado de sujetos con las mismas características analizables, y que pertenecen a una misma unidad de análisis.

La muestra de acuerdo a la fórmula del estadístico aleatorio simple fue de 70 Mypes. Según Otzen y Manterola (2017) a través de la muestra, la cual es extraída de la población, siendo posible extrapolarlas y como consecuencia, generalizar los resultados observados en dicha muestra.

$$
n=\frac{N * Z^{2} * P^{*} Q}{d^{2} *(N-1)+Z^{2} * P * Q}
$$

\section{Donde:}

$\mathrm{N}=$ Total de la Población (85 Pobladores)

$\mathrm{Z} \alpha=1,96$ al cuadrado (nivel de confianza al 95\%)

$\mathrm{P}=81 \%$ Probabilidad del éxito

$\mathrm{Q}=19 \%$ Probabilidad del Fracaso

$\mathrm{d}=$ Error de muestreo 5\%

\section{Reemplazando:}

$n=\frac{85 * 1.96^{2} * 0.81 * 0.19}{0.05^{2} *(85-1)+1.96^{2} * 0.81+0.19}=70$
$n \cong 70$


En ese sentido, Levine et al. (2007) refiere que, a efectos de realizar los análisis correspondientes en determinada población, será necesario hacer por cuestiones de presupuesto, la reducción a una muestra representativa de ésta, y estimar las características de dicha población. Para Espinoza (2013), a través del muestreo aleatorio simple, cada sujeto puede ser elegido bajo la misma probabilidad que los otros sujetos de estudio.

La técnica que se aplicó fue la encuesta y el instrumento fue el cuestionario. Al respecto Galindo (2000) refiere que la encuesta es una herramienta elemental en el estudio de las relaciones sociales. Por su parte, Namakforoosh (2005) manifiesta que los cuestionarios deben estar elaborados de tal manera que evidencien con facilidad y eficiencia lo que está sucediendo con las variables. En palabras de García (2004) el cuestionario es un sistema ordenado y racional de preguntas lógicas, expresadas en lenguaje comprensible.

En el procesamiento de la información se utilizó el software estadístico SPSS versión 26. Según Bausela (2005), el SPSS es una poderosa herramienta para el tratamiento de datos y el análisis de las estadísticas.

\section{RESULTADOS Y DISCUSIÓN}

\section{Información demográfica}

Según López (1998), las preguntas demográficas, por lo general, son parte de las investigaciones sociales, porque ofrecen a los investigadores información acerca de las edades, estado civil, condiciones que podrían proporcionarle detalles que otras preguntas no podrían lograr.

\section{Tabla 1}

Grupo Etario

\begin{tabular}{lcccc}
\hline & Frecuencia & Porcentaje & $\begin{array}{c}\text { Porcentaje } \\
\text { válido }\end{array}$ & $\begin{array}{c}\text { Porcentaje } \\
\text { acumulado }\end{array}$ \\
\hline de 21 a 30 años & 18 & 25.7 & 25.7 & 25.7 \\
de 41 a 50 años & 13 & 18.6 & 18.6 & 44.3 \\
más de 50 años & 39 & 55.7 & 55.7 & 100.0 \\
\hline Total & $\mathbf{7 0}$ & $\mathbf{1 0 0 . 0}$ & $\mathbf{1 0 0 . 0}$ & \\
\hline \hline
\end{tabular}

Nota: cuestionarios aplicados 2021.

De acuerdo a la Tabla 1, el mayor porcentaje de comerciantes $(55.7 \%)$ son mayores de 50 años. 


\section{Tabla 2}

Situación / condición del encuestado en la de la Asociación de Comerciantes Minoristas de la Plaza Internacional Túpac Amaru - ACOMITA II, del sector de venta de prendas de vestir.

\begin{tabular}{lcccc}
\hline & Frecuencia & Porcentaje & $\begin{array}{c}\text { Porcentaje } \\
\text { válido }\end{array}$ & $\begin{array}{c}\text { Porcentaje } \\
\text { acumulado }\end{array}$ \\
\hline Directivo & 12 & 17.1 & 17.1 & 17.1 \\
Asociado & 42 & 60.0 & 60.0 & 77.1 \\
Inquilino & 16 & 22.9 & 22.9 & 100.0 \\
\hline Total & $\mathbf{7 0}$ & $\mathbf{1 0 0 . 0}$ & $\mathbf{1 0 0 . 0}$ & \\
\hline \hline
\end{tabular}

De acuerdo a la Tabla 2, el mayor porcentaje de comerciantes que atienden y trabajan en el lugar son asociados $(60 \%)$.

Tabla 3

Años de experiencia en el negocio

\begin{tabular}{lcccc}
\hline & Frecuencia & Porcentaje & $\begin{array}{c}\text { Porcentaje } \\
\text { válido }\end{array}$ & $\begin{array}{c}\text { Porcentaje } \\
\text { acumulado }\end{array}$ \\
\hline Menos de 5 años & 12 & 17.1 & 17.1 & 17.1 \\
de 5 a 10 años & 32 & 45.7 & 45.7 & 62.9 \\
Más de 10 años & 26 & 37.1 & 37.1 & 100.0 \\
\hline Total & $\mathbf{7 0}$ & $\mathbf{1 0 0 . 0}$ & $\mathbf{1 0 0 . 0}$ & \\
\hline \hline
\end{tabular}

Nota: cuestionarios aplicados 2021.

De acuerdo a la Tabla 3, los comerciantes refieren tener, mayoritariamente (45.7\%) de 5 a 10 años de experiencia en sus actividades comerciales como Mypes.

\section{Tabla 4}

Nivel educativo

\begin{tabular}{lcccc}
\hline & Frecuencia & Porcentaje & $\begin{array}{c}\text { Porcentaje } \\
\text { válido }\end{array}$ & $\begin{array}{c}\text { Porcentaje } \\
\text { acumulado }\end{array}$ \\
\hline Primario & 26 & 37.1 & 37.1 & 37.1 \\
Secundaria & 26 & 37.1 & 37.1 & 74.3 \\
Superior & 18 & 25.7 & 25.7 & 100.0 \\
\hline Total & $\mathbf{7 0}$ & $\mathbf{1 0 0 . 0}$ & $\mathbf{1 0 0 . 0}$ & \\
\hline \hline
\end{tabular}

Nota: cuestionarios aplicados 2021.

De acuerdo a la Tabla 4, el nivel educativo de la mayoría de comerciantes es secundaria $(37.1 \%)$. 


\section{Tabla 5}

Género

\begin{tabular}{lcccc}
\hline & Frecuencia & Porcentaje & $\begin{array}{c}\text { Porcentaje } \\
\text { válido }\end{array}$ & $\begin{array}{c}\text { Porcentaje } \\
\text { acumulado }\end{array}$ \\
\hline Femenino & 58 & 82.9 & 82.9 & 82.9 \\
Masculino & 12 & 17.1 & 17.1 & 100.0 \\
\hline Total & 70 & 100.0 & 100.0 & \\
\hline \hline
\end{tabular}

Nota: cuestionarios aplicados 2021.

De acuerdo a la Tabla 5, el mayor porcentaje de comerciantes $(82.9 \%)$ pertenecen al género femenino.

\section{Análisis inferencial}

Con la finalidad de generalizar los resultados y obtener conclusiones atribuibles a la población, se hace necesaria la aplicación de estadígrafos inferenciales, es decir, hacer una estimación o inferencia; esto es importante, porque también se puede cuantificar la probabilidad del error al hacer esta estimación. En conclusión, el análisis inferencial permitirá extrapolar conclusiones. (Romero et al., 2018).

\section{Prueba estadística de hipótesis general}

H1: Los factores influyen significativamente en la liquidez de las Mypes de la plaza Túpac Amaru de la ciudad de Juliaca en épocas de Covid-19, en el periodo 2020.

H0: Los factores no influyen significativamente en la liquidez de las Mypes de la plaza Túpac Amaru de la ciudad de Juliaca en épocas de Covid-19, en el periodo 2020.

\section{Tabla 6}

Los factores influyen significativamente en la liquidez de las Mypes de la plaza Túpac Amaru de la ciudad de Juliaca en épocas de Covid-19, en el periodo 2020

\begin{tabular}{llcc}
\hline & & Factores & Liquidez \\
\hline \multirow{2}{*}{ Factores } & Correlación de Pearson & 1 &, $758^{* *}$ \\
& Sig. (bilateral) & & .000 \\
& $\mathrm{~N}$ & 70 & 70 \\
\hline \multirow{2}{*}{ Liquidez } & Correlación de Pearson &, $758^{* *}$ & 1 \\
& Sig. (bilateral) & .000 & 70 \\
\hline
\end{tabular}

**. La correlación es significativa en el nivel 0,01 (bilateral). Nota: cuestionarios aplicados 2021 
El valor del estadístico $r$ de Pearson es de 0.758, además esta correlación es muy significativa, por lo que se afirma con $99 \%$ de confianza que, en el ámbito del presente trabajo de investigación, hay una correlación positiva considerable entre la variable factores y la variable liquidez, porque el valor del Sig (bilateral) es de 0.000 que se encuentra por debajo del 0.01 requerido; entonces se acepta la hipótesis alternativa: Los factores influyen significativamente en la liquidez de las Mypes de la plaza Túpac Amaru de la ciudad de Juliaca en épocas de Covid-19, en el periodo 2020.

\section{Prueba estadística de hipótesis específica 1}

H1: El control de operaciones influye significativamente en la liquidez de las Mypes de la plaza Túpac Amaru de la ciudad de Juliaca en épocas de Covid-19, en el periodo 2020. H2: El control de operaciones no influye significativamente en la liquidez de las Mypes de la plaza Túpac Amaru de la ciudad de Juliaca en épocas de Covid-19, en el periodo 2020.

\section{Tabla 7}

El control de operaciones influye significativamente en la liquidez de las Mypes de la plaza Túpac Amaru de la ciudad de Juliaca en épocas de Covid-19, en el periodo 2020

\begin{tabular}{llcc}
\hline & & $\begin{array}{c}\text { Control de } \\
\text { operaciones }\end{array}$ & Liquidez \\
\hline \multirow{2}{*}{ Control de operaciones } & Correlación de Pearson & 1 &, $438^{* *}$ \\
& Sig. (bilateral) & & .000 \\
& $\mathrm{~N}$ & 70 & 70 \\
\hline \multirow{2}{*}{ Liquidez } & Correlación de Pearson &, $438^{* *}$ & 1 \\
& Sig. (bilateral) & .000 & \\
& $\mathrm{~N}$ & 70 & 70 \\
\hline
\end{tabular}

**. La correlación es significativa en el nivel 0,01 (bilateral).

Nota: cuestionarios aplicados 2021.

El valor del estadístico $\mathrm{r}$ de Pearson es de 0.438 , además esta correlación es positiva media, por lo que se afirma con $99 \%$ de confianza que, en el ámbito del presente trabajo de investigación, hay una correlación positiva media entre el control de operaciones y la liquidez, porque el valor del Sig (bilateral) es de 0.000 que se encuentra por debajo del 0.01 requerido; entonces se acepta la hipótesis alternativa: El control de operaciones influye significativamente en la liquidez de las Mypes de la plaza Túpac Amaru de la ciudad de Juliaca en épocas de Covid-19, en el periodo 2020. 


\section{Prueba estadística de hipótesis específica 2}

H1: El uso del registro de ingresos influye significativamente en la liquidez de las Mypes de la plaza Túpac Amaru de la ciudad de Juliaca en épocas de Covid-19, en el periodo 2020 .

H0: El uso del registro de ingresos no influye significativamente en la liquidez de las Mypes de la plaza Túpac Amaru de la ciudad de Juliaca en épocas de Covid-19, en el periodo 2020.

\section{Tabla 8}

El uso del registro de ingresos influye significativamente en la liquidez de las Mypes de la plaza Túpac Amaru de la ciudad de Juliaca en épocas de Covid-19, en el periodo 2020.

\begin{tabular}{llcc}
\hline & & Registro de ingresos & Liquidez \\
\hline \multirow{2}{*}{ Registro de ingresos } & Correlación de Pearson & 1 &, $857^{* *}$ \\
& Sig. (bilateral) & & .000 \\
& $\mathrm{~N}$ & 70 & 70 \\
\hline Liquidez & Correlación de Pearson &, $857^{* *}$ & 1 \\
& Sig. (bilateral) & .000 & 70 \\
\hline
\end{tabular}

**. La correlación es significativa en el nivel 0,01 (bilateral).

Nota: cuestionarios aplicados 2021.

El valor del estadístico $\mathrm{r}$ de Pearson es de 0.857 , además esta correlación es positiva muy fuerte, por lo que se afirma con 99\% de confianza que, en el ámbito del presente trabajo de investigación, hay una correlación positiva media entre el registro de ingresos y la liquidez, porque el valor del Sig (bilateral) es de 0.000 que se encuentra por debajo del 0.01 requerido; entonces se acepta la hipótesis alternativa: El uso del registro de ingresos influye significativamente en la liquidez de las Mypes de la plaza Túpac Amaru de la ciudad de Juliaca en épocas de Covid-19, en el periodo 2020.

\section{Prueba estadística de hipótesis específica 3}

H1: El uso del registro de gastos influye significativamente en la liquidez de las Mypes de la plaza Túpac Amaru de la ciudad de Juliaca en épocas de Covid-19, en el periodo 2020. 
H0: El uso del registro de gastos no influye significativamente en la liquidez de las Mypes de la plaza Túpac Amaru de la ciudad de Juliaca en épocas de Covid-19, en el periodo 2020.

\section{Tabla 9}

El uso del registro de gastos influye significativamente en la liquidez de las Mypes de la plaza Túpac Amaru de la ciudad de Juliaca en épocas de Covid-19, en el periodo 2020.

\begin{tabular}{llcc}
\hline & & $\begin{array}{c}\text { Registro de } \\
\text { gastos }\end{array}$ & Liquidez \\
\hline \multirow{2}{*}{ Registro de gastos } & Correlación de Pearson & 1 &, $933^{* *}$ \\
& Sig. (bilateral) & & .000 \\
& $\mathrm{~N}$ & 70 & 70 \\
\hline \multirow{2}{*}{ Liquidez } & Correlación de Pearson &, $933^{* *}$ & 1 \\
& Sig. (bilateral) & .000 & 70 \\
\hline
\end{tabular}

**. La correlación es significativa en el nivel 0,01 (bilateral).

Nota: cuestionarios aplicados 2021.

El valor del estadístico r de Pearson es de 0.933, además esta correlación es positiva muy fuerte, por lo que se afirma con 99\% de confianza que, en el ámbito del presente trabajo de investigación, hay una correlación positiva media entre el registro de gastos y la liquidez, porque el valor del Sig (bilateral) es de 0.000 que se encuentra por debajo del 0.01 requerido; entonces se acepta la hipótesis alternativa: El uso del registro de gastos influye significativamente en la liquidez de las Mypes de la plaza Túpac Amaru de la ciudad de Juliaca en épocas de Covid-19, en el periodo 2020.

\section{Prueba estadística de hipótesis específica 4}

H1: La elaboración de los estados financieros influyen significativamente en la liquidez de las Mypes de la plaza Túpac Amaru de la ciudad de Juliaca en épocas de Covid-19, en el periodo 2020 .

H0: La elaboración de los estados financieros influyen significativamente en la liquidez de las Mypes de la plaza Túpac Amaru de la ciudad de Juliaca en épocas de Covid-19, en el periodo 2020 . 


\section{Tabla 10}

La elaboración de los estados financieros influyen significativamente en la liquidez de las Mypes de la plaza Túpac Amaru de la ciudad de Juliaca en épocas de Covid-19, en el periodo 2020.

\begin{tabular}{llcc}
\hline & & $\begin{array}{c}\text { Estados } \\
\text { financieros }\end{array}$ & Liquidez \\
\hline \multirow{2}{*}{ Estados financieros } & Correlación de Pearson & 1 &, $267^{*}$ \\
& Sig. (bilateral) & & .025 \\
& $\mathrm{~N}$ & 70 & 70 \\
\hline Liquidez & Correlación de Pearson &, $267^{*}$ & 1 \\
& Sig. (bilateral) & .025 & 70 \\
\hline
\end{tabular}

*. La correlación es significativa en el nivel 0,05 (bilateral).

Nota: cuestionarios aplicados 2021.

El valor del estadístico r de Pearson es de 0.267, además esta correlación es positiva débil, por lo que se afirma con $95 \%$ de confianza que, en el ámbito del presente trabajo de investigación, hay una correlación positiva débil entre los estados financieros y la liquidez, porque el valor del Sig (bilateral) es de 0.000 que se encuentra por debajo del 0.05 requerido; entonces se acepta la hipótesis alternativa: La elaboración de los estados financieros influyen significativamente en la liquidez de las Mypes de la plaza Túpac Amaru de la ciudad de Juliaca en épocas de Covid-19, en el periodo 2020.

\section{DISCUSIÓN}

A efectos de cumplir con los objetivos del presente artículo, se llevaron a cabo encuestas, las mismas que, en relación a los factores que influyen en la liquidez de las Mypes, este trabajo concluye que existe una relación muy significativa entre las variables propuestas. Existen factores que influyeron en la liquidez de los microempresarios de la unidad de análisis.

En concordancia con Torres y Fernández (2021) quien concluyó que el crecimiento no será mayor al 7,9\%, así mismo refirió que la recuperación tardaría hasta el año 2023 o 2024, en ese sentido, Blanco et al. (2020) concuerda con la postura de este trabajo de investigación, al sostener el impacto negativo de la pandemia sobre las actividades económicas y los ingresos de los empresarios, provocando moratorias en sus compromisos de pagos. 
Respecto a los objetivos específicos, en relación al control de operaciones se evidencia que un $25.7 \%$ sí lleva un control de sus operaciones, un $25.7 \%$ lo hace esporádicamente, pero un $48.6 \%$ no lo sí lleva un control de todas sus operaciones comerciales.

Esto evidencia que el modelo económico y tributario no tiene llegada a las Mypes del interior del país, por lo que se infiere que, probablemente, la pandemia no fue el problema, el problema está en la estructura planificada por el Estado peruano, que solamente sirve en tiempos de bonanza, con el atenuante de que el sistema no se preocupa por capacitar al micro empresario a efectos de su trascendencia en el ámbito empresarial, por el contrario, su preocupación estriba en imposición de multas y sanciones o en la recaudación de impuestos, inclusive de manera coercitiva (Zevallos, 2018).

El segundo objetivo específico refiere a los registros de ingresos, los cuales, contablemente, son de vital importancia a efectos de controlar la rotación de la mercadería y el control de los stocks, éste se relaciona positiva y fuertemente con la liquidez, al respecto y concordando con Gitman y Joehnk (2009) quienes sostienen que la liquidez es el resultado de la capacidad que posee el empresario para atender a sus necesidades diarias, ello requiere de un orden y control de las existencias, su rotación y su valuación. Sin embargo, la realidad de las Mypes del presente estudio, es que no hay un control al respecto, ello concuerda con el estudio de Huayamave (2018) quien en su estudio concluyó que el sistema contable es deficiente, lo que ocasiona la debilidad de sus operaciones.

El tercer objetivo específico está relacionado con el registro de gastos, el cual tiene una relación positiva y fuerte, sin embargo, su uso no está plenamente identificado dentro de estas Mypes en estudio, lo cual podría evidenciar la erogación del efectivo en actividades que no reúnen las características de fuente de renta. El empresario, movido por su interés de permanecer en el mercado y llevar el sustento a sus familias, lleva un control de sus ingresos, utilizando la partida simple, dicha práctica se extiende también al control de sus gastos; sin embargo, en el presente estudio se ha encontrado que las Mypes de la unidad de análisis, en un $51.4 \%$ sí llevan el control de sus ingresos, pero solamente un $38.6 \%$ lleva un control de sus gastos.

A decir de esto, la Administración tributaria, la Cámara de Comercio, las Universidades y otros entes del sector, debieran procurar asistencias, capacitaciones respecto de sus necesidades, puesto que un verdadero control de las operaciones e información real y 
oportuna, se traduce en una buena toma de decisiones a efectos de preservar la liquidez durante todos los tiempos, incluyendo los de pandemia (Amat, 2008). Concordando con ello, Sánchez (2021) concluyó que no se está llevando un adecuado análisis y control de los desembolsos que se realizan, esto puede mermar las utilidades.

El cuarto objetivo específico refiere la importancia de los estados financieros, los mismos que, desde el punto de vista contable, son de vital importancia para muchos efectos tales como la toma de decisiones, la conservación de la liquidez, entre otros. Los comerciantes de las Mypes de la unidad de análisis no consideran necesaria la revisión de sus estados financieros, por diferentes razones: por desconocimiento de ellas, porque sus asesores contables no les proveen dicha información, o porque no saben interpretarla, o porque sencillamente no tienen la información requerida a fin de generarlas.

Una vez más se cita a los entes rectores del sector, llámese Ministerio de la Producción, Administración Tributaria, Cámara de Comercio, Gerencia de Desarrollo Económico del GORE y de las municipalidades, incluidas las universidades e institutos a quienes concierne este asunto. Aquí son elementales las capacitaciones por parte de las entidades aludidas, a fin de proveer de más y mejores herramientas de control operacional en estos tiempos de virtualidad (Correa, 2018). Al respecto, el estudio de Huapaya y Melendez (2017) concluyó que los estados financieros son importantes, puesto que influyen positivamente en la competitividad de las empresas, en las decisiones de inversión y en la toma de decisiones.

\section{CONCLUSIÓN O CONSIDERACIONES FINALES}

La primera conclusión a la que se llegó está relaciona con el objetivo general, determinar si los factores influyen en la liquidez de las Mypes en tiempos de pandemia por la Covid19. Dichos factores son extraídos de la variable uno y se traducen en información de calidad, e información oportuna.

Con respecto al primer objetivo específico, se concluye que existe una relación positiva media entre el control de operaciones y la liquidez, en contexto, el emprendedor de la Mypes debe tomar conciencia de que el azar, la suerte, los buenos tiempos y otros sucesos fortuitos, son solo eso, hechos ocurridos por ventura. El aseguramiento de la liquidez requiere del control de operaciones, pero de uno específico, ordenado y metódico dentro de las Mypes, ello asegurará un flujo constante de liquidez para poder cumplir con las obligaciones contraídas. 
Con respecto al segundo objetivo específico, se concluye que el registro de ingresos se relaciona con la liquidez de manera positiva y fuerte. En el contexto del presente trabajo, se pudo identificar ciertas habilidades en los empresarios de las Mypes de estudio, sin embargo, la información contable específica, es decir, la de los ingresos debe ser convertirse en habitual dentro de la unidad de análisis.

Respecto al tercer objetivo específico, se concluye que el registro de gastos tiene relación positiva muy fuerte con la liquidez, ello confirma la hipótesis contable del control de ingresos y gastos para asegurar el control de las operaciones y, sobre todo, la liquidez a efectos de minimizar la merma del efectivo en adquisiciones innecesarias.

El cuarto objetivo específico concluye que existe una relación positiva débil entre los estados financieros y la liquidez, lo que significa que a pesar de la importancia contable de dichos estados, éstos podrían prescindirse dentro de las Mypes de estudio, sin embargo, quedó claro que el líquido elemento no es producto del azar o de una buena racha, más bien, obedece a una buena planificación en base a información de calidad cuya forma y modo se encuentra instituida en las verdaderas prácticas contables.

\section{LISTA DE REFERENCIAS}

Amat i Salas, O. (2008). Análisis integral de empresas (B. E. S.L. (ed.); Bresca Edi).

Bárcena, A., (2020). Sectores y empresas frente al COVID-19: emergencia y reactivación.

Barco, E. (2021). Neuromarketing y publicidad para las pequeñas empresas emergentes del Emprio comercial de Gamarra, 2020 (UCV).

Bausela, E. (2005). Spss: Un Instrumento De Análisis De Datos Cuantitativos. Revista de Informática Educativa y Medios Audiovisuales, 2(4), 62-69.

Blanco, R., Mayordomo, S., Menéndez, Á., y Mulino, M. (2020). Las necesidades de liquidez y la solvencia de las empresas no financieras españolas tras la perturbación del Covid-19. Documentos Ocasionales, 2020.

Carbó, S. (2020). Por la Recuperación Financiera de las Empresas tras el COVID-19. Fedea Policy Papers - 2020. https://ec.europa.eu/competition/state_aid/scoreboard/index_en.html

Correa, F. (2018). Caracterización de la capacitación y la competitividad en las MYPE del rubro restaurantes de la urbanización Sant Ana de Piura, año 2018. Tesis de grado, Universidad Católica Los Ángeles de Chimbote.

Espinoza, I. (2013). Tipos de muestreo. Academia, 3-7. 
Galindo, L. (2000). Técnicas de investigación en sociedad, cultura y comunicación (Prentice

$\mathrm{H})$. https://books.google.com.pe/books?id=5a0Jdv7Ip9oC\&printsec=frontcover\&hl= es\# $\mathrm{v}=$ onepage $\& \mathrm{q} \& \mathrm{f}=$ false

García, F. (2004). El cuestionario: recomendaciones metodológicas para el diseño de cuestionarios (LIMUSA).

Gitman, L., y Joehnk, M. (2009). Fundamentos de inversiones (Pearson, A). https://books.google.es/books?hl=es\&lr=\&id=lTMNR9MUjuAC\&oi=fnd\&pg=P $\mathrm{R} 11 \& \mathrm{dq}=$ fundamentos + de + inversiones $+\&$ ots $=\mathrm{A} 6$ DrCLMn3O\&sig $=\mathrm{WkEmfbcq}$ nxc dUDXjMImGY-5fSh0\#v=onepage $\& \mathrm{q}=$ fundamentos de inversiones $\& \mathrm{f}=$ false

Hernández, B. (2018). Técnicas estadísticas de investigación social. https://books.google.com.pe/books?id=vpfVgmaR5qUC\&printsec=frontcover $\&$ $\mathrm{dq}=$ población $+\mathrm{y}+$ muestra $+\mathrm{de}+$ la + investigación \&hl=es419\&sa=X\&ved=2ahUK Ewi5uKw4a3tAhWlH7kGHT7RA80Q6AEwAHoECAEQAg\#v=onepage\&q=po blació y muestra de la investigación\&f=false

Hernández Sampieri, R., y Mendoza Torres, C. P. (2018). Metodología de la investigación: las tres rutas cuantitativa, cualitativa y mixta. In Mc Graw Hill (Vol. 1, Issue Mexico).

Huapaya, S., y Melendez, E. (2017). Los estados financieros y su importancia en la toma de decisiones en las empresas comerciales en la provincia de Huaura 2016 (Vol. 0). Tesis de grado, Universidad Nacional Jospe Faustino Sánchez Carrión.

Huayamave, J. (2018). Propuesta de mejoras al registro de ingresos y egresos en la asociación agrícola ganadera Rio Daule. Tesis de grado, Instituto Superior Tecnológico Bolivariano de Tecnología.

Levine, D., Krehbiel, T., y Berenson, M. (2007). Estadística para la administración (Pearson Ed).

López, H. (1998). La metodología de encuesta. Técnicas de Investigación En Sociedad, Cultura $y$ Comunicación., 33-73. https://biblioteca.marco.edu.mx/files/metodologia_encuestas.pdf

Namakforoosh, M. (2005). Metodología de la investigación (Limusa).

Otzen, T., y Manterola, C. (2017). Técnicas de Muestreo sobre una Población a Estudio. Scielo - International Journal of Morphology, 35(1), 227-232. 
https://doi.org/10.4067/S0717-95022017000100037

Ramos, C. (2020). Los Alcances de una investigación. CienciAmérica, 9.

Romero, P., Lázaro, C., y Gonzáles, J. (2018). Estadística descriptiva e inferencial. Estadística, January 2013, 1-280.

Sánchez, M. (2021). El principio de causalidad en los gastos de la empresa La Taberna distribuciones SAC - 2018. Tesis de grado, Universidad Señor de Sipán.

Torres, P. (2016). Acerca de los enfoques cuantitativo y cualitativo en la investigación educativa cubana actual. Atenas Revista Cietífico Pedagógica, 2(34), [1-15]. http://atenas.umcc.cu/index.php/atenas/article/view/194/363\%0Ahttp://atenas.u mccu/index.php/atenas/article/view/194

Torres, R., y Fernández, M. (2021). La política económica española y el COVID-19. Cuadernos de Información Económica, 1-7.

Torres, R., Fernández, M., y Maudos, J. (2020). Cuadernos de Información Económica. Cuadernos de Información Económica, 278, 1-69.

Zevallos, P. (2018). La SUNAT y la defraudación tributaria en el Perú. Tesis de grado. Universidad de Huánuco. 